\title{
Cultural adaptation of an olfactory test: the odour in bottle
} test*

\author{
Aylin Gül, Mehmet Akdağ, Fazıl Emre Özkurt, Beyhan Yılmaz, Engin Şengül, \\ Salih Bakır, İsmail Topçu \\ Rhinology 52: 172-177, 2014 \\ DOl:10.4193/Rhino13.055 \\ *Received for publication: \\ May 6, 2013 \\ Department of Otorhinolaryngology, Dicle University School of Medicine, Diyarbakir 21280, Turkey
}

Accepted: December 13, 2013

\begin{abstract}
Aim: Our objective was to create a culturally appropriate test of olfactory perception based on the Sniffin' Sticks test to determine the effects of age, gender, education level, and smoking on patients' sense of smell in Diyarbakır, Turkey.

Material and Methods: A total of 180 subjects participated in the study including 111 males. These were patients at the Dicle University Otolaryngology Polyclinic and voluntarily participated in the study between June and December 2012. They were subdivided according to age: Group 1 included 100 patients between 18-35 years of age, Group 2 contained 50 patients between 36-55 years of age, and Group 3 was comprised of 30 patients over 55 years old. All subjects received olfactory testing with our modified Sniffin' Sticks test.
\end{abstract}

Results: There was a significant negative correlation between olfactory perception scores and increasing age. No significant relationship was found between olfactory perception and gender. When olfaction scores were evaluated according to education level, it was found that subjects with lower education had significantly decreased olfaction scores. Smokers also had significantly lower olfactory perception scores when compared to nonsmokers. In terms of odorant identification, sesame and cumin were the least likely to be correctly identified, as they were recognized only $21 \%$ and $40 \%$ of the time, respectively.

Conclusion: We created a culturally appropriate test of olfactory perception based on the Sniffin' Sticks test to determine the effects of age, gender, education level, and smoking on our local patients' sense of smell. Our results suggest that age and smoking status negatively affect olfaction, and cumin and sesame should be replaced by more culturally familiar odorants.

Key words: identification, discrimination, threshold, Sniffin'Sticks, olfactory test, odorant

\section{Introduction}

Currently there are no methods to objectively evaluate olfactory dysfunction. Although olfactory testing is not routinely performed in the clinical setting, neglecting to identify olfactory dysfunction may have serious health implications ${ }^{(1)}$. Several tests have been developed to examine the human sense of smell, and the University of Pennsylvania Smell Identification Test is most commonly utilized in health care settings ${ }^{(2)}$. Another olfactory screening modality is the Zurich Smell Diskette, which is particularly useful as it has both visual and olfactory components. The
Barcelona Smell Test-24 (BAST-24) is widely used to test olfaction in patients from Spain ${ }^{(3)}$. In North America, the Connecticut Chemosensor and Clinical Research Center (CCCRC) test is the most commonly used test, whereas the Sniffin' Sticks test is usually utilized in Europe and Australia ${ }^{(2,4)}$. The CCCRC test has been developed previously by the authors. It is a validated and well described test ${ }^{(5)}$.

The Sniffin' Sticks (Heinrich Burghart GmbH, Wedel, Germany) test is conducted with pen-like odorant materials and consists of three subtests: olfactory threshold (OT), odour discrimination 
(OD), and odour identification (OI). The OT subtest measures how sensitive a patient's sense of smell is by exposing him/her to low concentration scents. The OD subtest measures a patient's ability to discriminate between different odours, and the Ol subtest determines whether a patient can correctly identify certain odours. Clinical efficacy of the Sniffin' Sticks test for the European patient population was published in European Position Paper on Rhinosinusitis and Nasal Polyps (EPOS) in $2012^{\text {(3). }}$ So far, the Sniffin' Sticks test has been utilized by many clinicians throughout the world and has been approved for use in various countries including Australia, Greece, Taiwan, Italy, the Netherlands, Sri Lanka, and Brazil ${ }^{(6-13)}$.

To test for olfactory dysfunction accurately, the test must be adapted such that the scents used are familiar to the patient population that is being tested. As such, it is imperative that tests for olfactory dysfunction are designed in a manner that is culturally appropriate. In this study, we aimed to evaluate olfaction and correlate our findings with subject age, gender, smoking, and education levels by selecting odorants that were culturally familiar to patients living in Diyarbakır, Turkey.

\section{Materials and methods}

\section{Ethical considerations}

All subjects were informed that participation was completely voluntary, and they were given a detailed explanation of the entire study protocol. Voluntary consent was obtained from all the study participants. The study was approved by the departmental ethics committee and was carried out in accordance with the Declaration of Helsinki as amended in 2008 (08.10.2012/167).

\section{Study subjects}

A total of 180 patients that presented to the Dicle University Otolaryngology Polyclinic between June and December 2012 with a variety of chief complaints were enrolled into the study. Of the subjects in the patient sample, 111 were male, and all patients received routine otolaryngology testing. Exclusion criteria for study participation were patients under 18 years; having a history of deviated nasal septum, concha hypertrophy, allergic rhinitis, nasal polyps, or sinusitis; having a history of head or neck trauma; having a neurodegenerative illness; and having

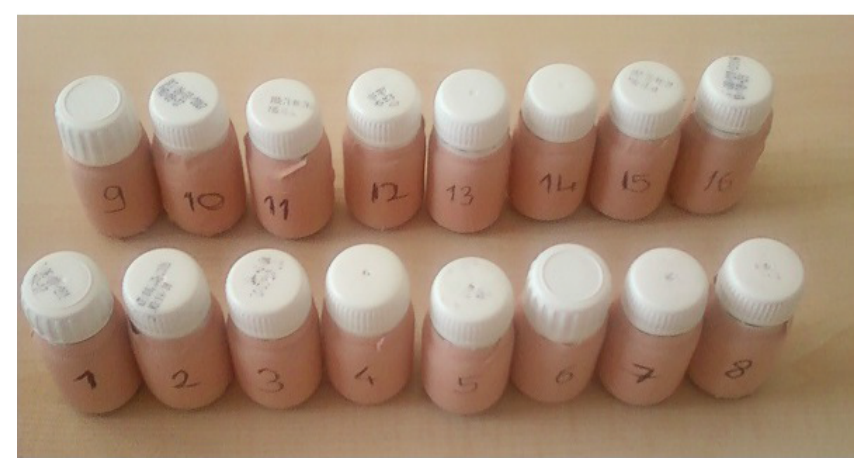

Figure 1. Sixteen different odorants tested in the study.

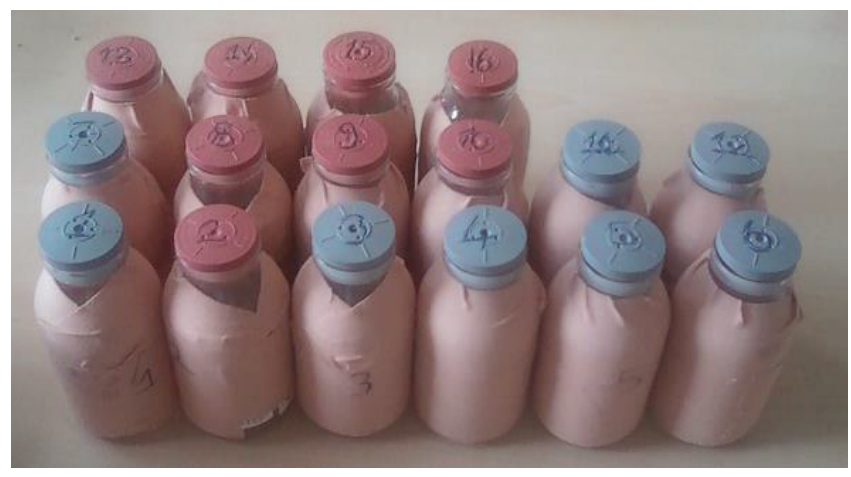

Figure 2. Sixteen bottles of diluted n-butanol.

a history of upper respiratory infection within the last 10 days of study participation. Study subjects were divided into groups based on age (Table 1). Group 1 consisted of patients from 18 to 35 years old ( $n=100)$, Group 2 was comprised of patients ranging from 36 to 55 years old $(n=50)$, and Group 3 included patients older than 56 years of age $(n=30)$. Patients were then further subdivided into three groups according to education level: Group 1 included patients that had graduated from primary school $(n=64)$, Group 2 was comprised of subjects that graduated from high school $(n=74)$, and Group 3 had patients that graduated from college $(n=42)$.

\section{The bottle odour test}

Sixteen different odorants that were considered culturally appropriate for the patient sample were selected (Figure 1). These

Table 1. Demographic characteristics of patients groups.

\begin{tabular}{|c|c|c|c|c|c|c|c|c|}
\hline Parameter & $18-35$ years & $36-55$ years & over 55 years & $\begin{array}{c}\text { Primary } \\
\text { school }\end{array}$ & High school & College & Smoker & Non-smoker \\
\hline Female & 42 & 14 & 13 & 22 & 29 & 18 & 10 & 59 \\
\hline Male & 58 & 36 & 17 & 42 & 45 & 24 & 75 & 36 \\
\hline Total & 100 & 50 & 30 & 64 & 74 & 42 & 85 & 95 \\
\hline
\end{tabular}


odorants were aromatic herbal oils and were acquired from an herb and spice seller (Talya Herbal Products). Odorants that were chosen for the study were cinnamon, apple, rose, lemon, thyme, garlic, cloves, cumin, black pepper, sesame, menthol, mint, orange, coconut, coffee, and fish. These aromatic herbal oils were kept in separate bottles and were synthesized from $100 \%$ natural materials. We designed this test such that it would be comparable to the Sniffin' Sticks test with OT, OD, and OI subtests. After each subject completed all three subtests, the results were compiled and presented as the TDI score.

\section{Odour identification}

Subjects were presented with the entire set 16 different odours in $20 \mathrm{~mL}$ bottles that were $5 \mathrm{~cm}$ tall and $2 \mathrm{~cm}$ in diameter (Figure 1). The subjects were instructed to hold the bottle for 3 seconds at a distance of $2 \mathrm{~cm}$ in front of the nose such that the bottle was aligned with the patient's midline. The subject was told to smell each of the 16 bottles one at a time and then they were asked to identify each odorant and mark the corresponding scent on an identification list (Table 2). The odorants were presented at intervals of 20 to 30 seconds. A score of zero was awarded for an incorrect answer. The highest total score that could be attained was 16 points.

\section{Odour discrimination}

Three bottles were prepared randomly such that the same odorant was in two different bottles and another odorant was in the third bottle. The subject then smelled all three odorants and then the subject was asked to identify the odorant that was different. Ten seconds were given between odours within the same odorant set, and the subject was allowed to smell each odorant for approximately 3 seconds. Different sets of three odorants were presented at intervals of 20 to 30 seconds. A total of 16 tests were performed with 1 point awarded for each correct answer.

\section{Odour threshold}

The odorant $n$-butanol was utilized to determine odour threshold. Solutions of $4 \% \mathrm{n}$-butanol were diluted serially at a 1:2 ratio with distilled water (Figure 2). The bottles utilized for this test contained $100 \mathrm{~mL}$ of odorant and were $10 \mathrm{~cm}$ tall and $3 \mathrm{~cm}$ in diameter. Each subject smelled each bottle for 3 seconds and every odorant was presented at 10-second intervals. The odour threshold test was administered by a single doctor with no diagnosed olfactory dysfunction, who reported that his sense of smell was not impaired in the well air-conditioned examination room. Before each test was conducted, the physician would evaluate whether the odorant could be clearly identified. If the odorant could not be smelled by the physician, then the bottle was discarded and replaced with a backup bottle of the same concentration to ascertain whether the odorant could be
Table 2. Odour identification list.

\begin{tabular}{|c|c|c|c|c|}
\hline 1 & Cinnamon & Sesame & Lemon & Coffee \\
\hline 2 & Cloves & Garlic & Apple & Sesame \\
\hline 3 & Coffee & Banana & Rose & Cloves \\
\hline 4 & Lemon & Cinnamon & Banana & Strawberry \\
\hline 5 & Cloves & Banana & Thyme & Coconut \\
\hline 6 & Lemon & Garlic & Black pepper & Vinegar \\
\hline 7 & Orange & Cloves & Thyme & Rose \\
\hline 8 & Thyme & Apple & Cumin & Cinnamon \\
\hline 9 & Vinegar & Coffee & Banana & Black pepper \\
\hline 10 & Garlic & Cloves & Menthol & Sesame \\
\hline 11 & Menthol & Coffee & Rose & Cloves \\
\hline 12 & Banana & Black pepper & Mint & Sesame \\
\hline 13 & Orange & Coconut & Cinnamon & Thyme \\
\hline 14 & Rose & Lemon & Apple & Coconut \\
\hline 15 & Cloves & Coffee & Cinnamon & Cumin \\
\hline 16 & Garlic & Fish & Vinegar & Black pepper \\
\hline
\end{tabular}

detected. Three bottles of varying $\mathrm{n}$-butanol concentrations were presented to the subject randomly and the subject was to determine which bottle contained an odorant. Odour thresholds for $n$-butanol was assessed using a single-staircase, triple-forced choice procedure. If the odour was detected correctly in two tests in series, then the staircase system was cancelled. Each test took approximately 15 minutes to administer. A total of 20 seconds was allowed between each odorant. The same 16-point scoring system as utilized for this test.

\section{Statistical analyses}

All the data were organized and analyzed by SPSS version 15.0 for Windows (SPSS Inc, Chicago, IL, USA). Average and frequency distributions were examined to determine whether the data followed a normal distribution. For data that were normally distributed, Student's t-test and one-way ANOVA were utilized to analyze the data. For data that did not conform to a normal distribution, the Kruskal-Wallis test and Mann-Whitney U-test were applied. A p-value less than 0.05 was considered statistically significant.

\section{Results}

Average total scores for the OT, OD, and Ol subtests were 6.5 ( \pm $2.5), 11.5( \pm 2.7)$, and $11.4( \pm 2.7)$, respectively. The average total 


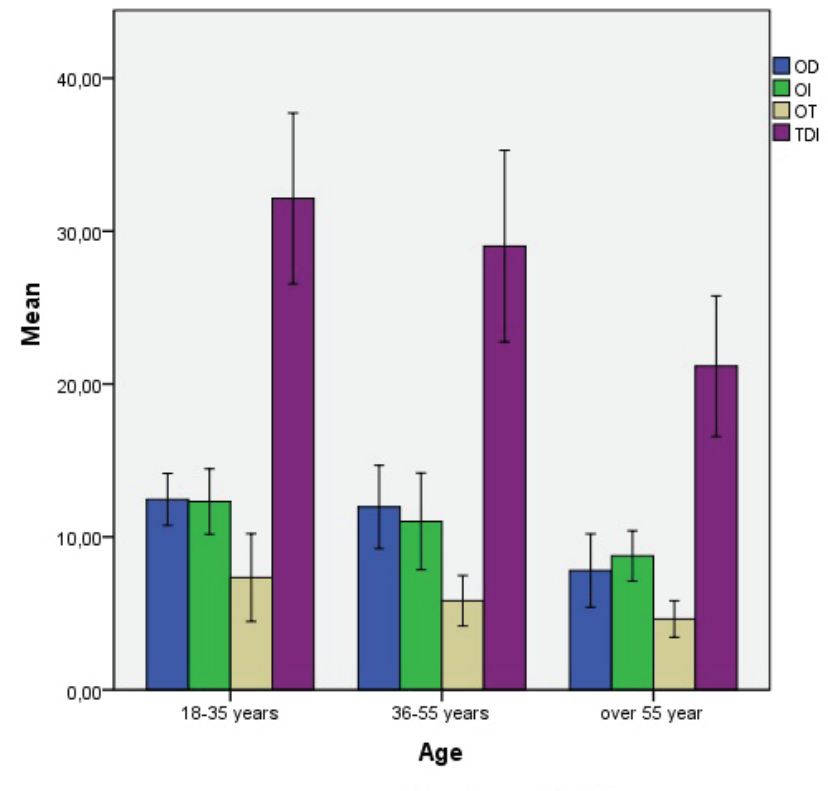

Figure 3. Relationship between olfactory scores and age. Group 1 and group 2 in OD was seen statistically nonsignificant $(p>0.05)$. Other comparisons: statistically significant, $(p<0.01)$.

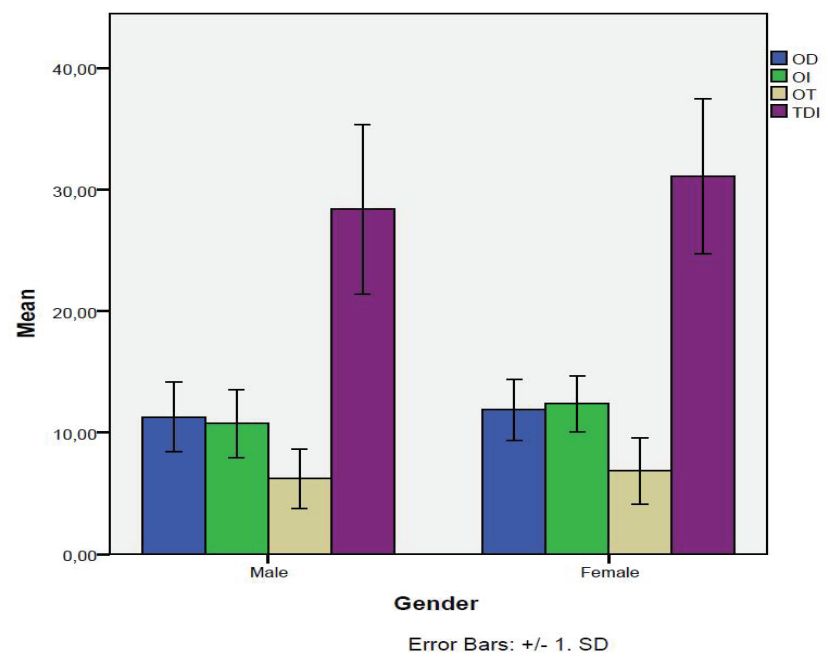

Figure 4. Relationship between olfactory scores and gender. OI and TDI scores were statistically significant $(p<0.01)$.

score that included all subtest scores, also known as the TDI, was $29.3( \pm 6.9)$.

\section{Age}

The average age of the entire patient sample was $38.3( \pm 11.8)$ years. The oldest female participant was 62 years old, and the oldest male subject was 66 years old. A statistically significant decrease in TDI and subtest scores was observed with increasing age $(p<0.01)$. However, no difference was found between groups 1 and 2 for OD ( $p>0.05$; Figure 3 ).

\section{Gender}

The average ages for the female and male cohorts were 35.1 and 41.5 years, respectively. No statistically significant differences were observed between gender for the OT and OD subtest scores $(p>0.1)$. However, there was a significant difference in scoring for the OI and TDI subtests according to gender ( $p<$ 0.01; Figure 4).

\section{Education Level}

The study subjects were assigned to three groups according to their educational level. Comparing the subjects based on their educational background, it was determined that the average OT subtest scores differed significantly between these groups. A significant difference was observed between Groups 1 and 2 and Groups 1 and 3 for average OD scores, but no significant difference was observed between Groups 2 and $3(p=0.02)$. A statistically significant difference was found between all three groups for average OI scores and average TDI scores $(p<0.01$; Figure 5).

\section{Use of Cigarettes}

A total of 85 study subjects smoked between 1 and 50 packs of cigarettes per year, and the average number of cigarette packs smoked per year was 20.35 packs. No statistically significant difference were observed between smokers and the nonsmokers in terms of the OD subtest scores ( $p>0.1)$. Yet, statistically significant differences were observed between smokers and nonsmokers for the OT, OI, and TDI scores ( $p<0.01$ ).

In terms of odorant identification, sesame and cumin were least likely to be correctly identified, as they were recognized only $21 \%$ and $40 \%$ of the time, respectively. However, mint and coffee were most likely to be correctly identified at $96.1 \%$ and $97.8 \%$ of the time in that order (Figure 6).

\section{Discussion}

Olfactory perception begins to decrease at 65 years of age and older ${ }^{(14)}$. This decrease in sensitivity to smell with increasing age is due to changes in nerve connections and cortical pathways, decreasing blood flow to the olfactory area in the brain, increasing mucus viscosity, decreasing overall metabolic activity, and psychological factors ${ }^{(6)}$. Thus, age is certainly a relevant contributor to olfactory dysfunction ${ }^{(9,15)}$. In a study with a sample size of 1,955 subjects by Doty et al., it was found that major olfactory deficits occurred in $50 \%$ of subjects that were $65-80$ years old and in $75 \%$ of subjects 80 years and older ${ }^{(16)}$. Hummel et al. reported that OT decreases more drastically than either the Ol or the OD with increasing age ${ }^{(17)}$. In our study, the average participant age was 38.3 ( \pm 11.8 ) years, and our results revealed important decreases in all OT, OD, OI subtest scores and TDI scores at older ages. 


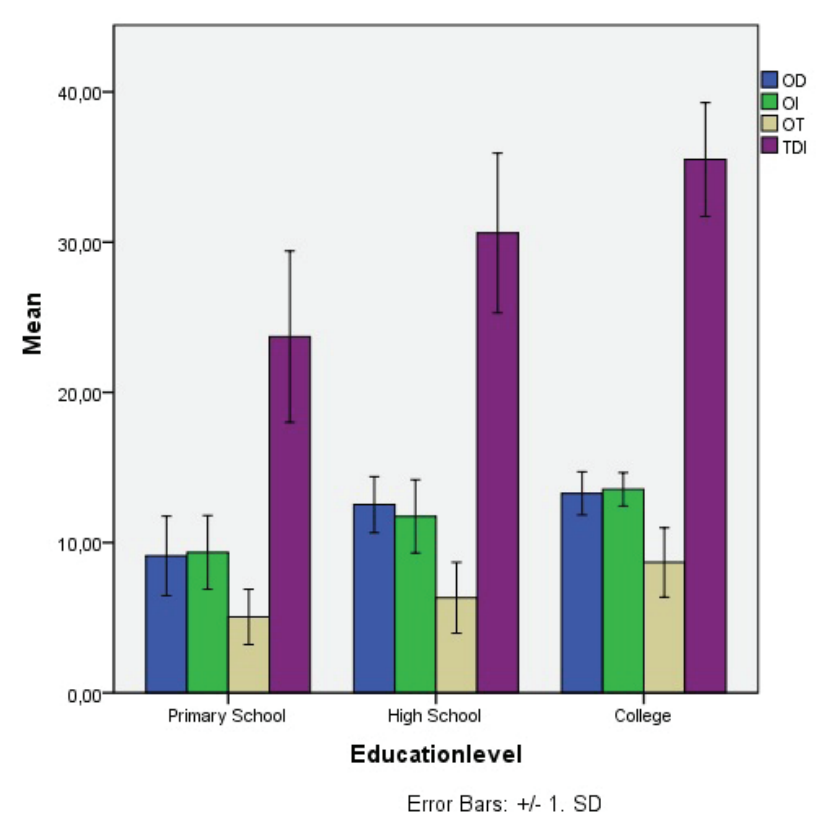

Figure 5. Relationship between olfactory scores and education. Group 2 and group 3 OD was seen statistically nonsignificant $(p=0.02)$. Other comparisons: statistically significant, $(p<0.01)$.

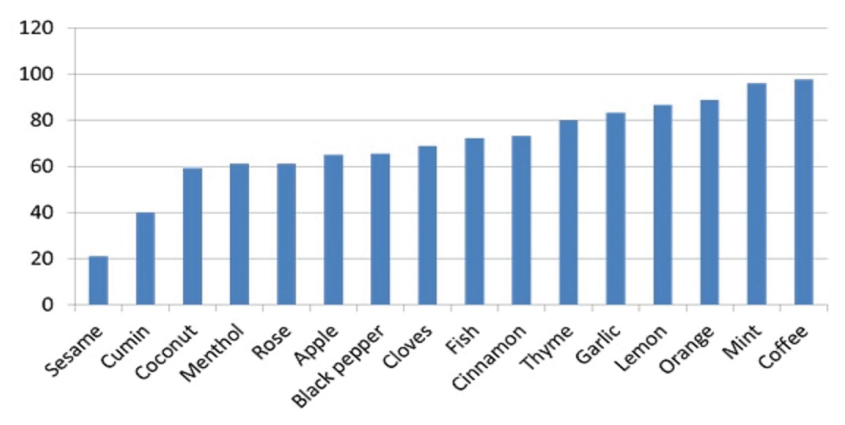

Figure 6. Identification ratios of all odours.

Currently, there is little understanding regarding how hormones regulate the human sense of smell ${ }^{(18)}$. Katotomichelakis et al. demonstrated that OT and TDI scores were significantly higher in females when compared to males ${ }^{(6)}$. However, Hummel et al. reported that there were no significant differences between male and female TDI scores in a group of consisting of 3,000 patients ${ }^{(17)}$. Orhan et al. also found that females scored higher, but this difference was not found to be statistically significant ${ }^{(18)}$. In our study, the effect of gender on the OT and OD subtest scores were not statistically significant. However, females had significantly higher OI and TDI scores.

When subject education levels were compared, the scores for those with lower education levels were significantly lower than those with moderate or higher education levels. Yet, when educational background was evaluated in terms of age, subjects with lower education levels were older than their more educa- ted counterparts. As such, it was not possible to draw any correlations between decreased scores and education levels in our study. Similarly, Orhan et al. reported an association between lower education and decreased scores ${ }^{(18)}$.

The negative effects of smoking on olfactory function are manifest over time. Cigarette chemicals that are carried in air into the nasal cavity while smoking affect the olfactory receptors and apoptosis increases in olfactory sensory neurons ${ }^{(19)}$. The detrimental effects of smoking on olfactory perception are still debated as various studies propose that the compromise in olfaction is permanent while other studies assert that its negative effects are transient ${ }^{(20-23)}$. Katotomichelakis et al. demonstrated via multivariable linear regression analysis that the effects of smoking are independent of gender and age, and they showed that olfactory sensitivity is negatively correlated with smoking (19). However, Orhan et al. did not detect a relationship between smoking and olfactory scores in their study ${ }^{(18)}$. Nevertheless, we found that smokers had scored lower in their olfactory testing in contrast with the nonsmokers.

Though many techniques exist to examine olfactory functioning, the extended version of the Sniffin'Sticks test has been deemed to be superior to other olfactory tests for determining OT, OD, OI, and TDI (24). Our study utilized a modified version of the Sniffin' Sticks test as we utilized odorants that were deemed to be culturally familiar to the our geographical region patients' population tested in this investigation. It is important to cater odorant selection based on cultural background, as was demonstrated in the study performed by Orhan et al., as they determined that their patient sample was less likely to correctly identify turpentine, licorice, and apple. They observed that their subjects often mistook the apple odorant for the scent of peaches. Many participants stated that they were not familiar with the scents of turpentine, licorice, and aniseed and the participants suggested that these odours should be replaced with more familiar ones ${ }^{(18)}$. In response to this suggestion, we endeavored to create a battery of odorants that were most familiar with the patient sample that we worked with based on the work by Orhan et al. We did not include turpentine, licorice, aniseed, cloves, and the deceiving identifiers for apple were changed to garlic and sesame. However, we found that that sesame and cumin were not correctly identified most frequently. These results may be due to the fact that sesame is not commonly encountered by the patient sample that we studied. Interestingly, cumin is a commonly used spice in the region where the population sample was derived. We attributed patient's inability to identify cumin as other erroneous scent choices distracted the subjects from recognizing this odorant. For future studies, we suggest replacing sesame and cumin with even more easily identifiable odorants. Neumann et al. suggested that deceiving odorant 
identifiers should be changed for scents that have particularly low correct identification rates ${ }^{(25)}$. However, there are two studies that we are planning to conduct in the near future. In those studies, we will utilize the Sniffin' Sticks test and CCCRC test.

\section{Conclusion}

We created a culturally appropriate test of olfactory perception based on the Sniffin' Sticks test to determine the effects of age, gender, education level, and smoking on our geographical region patients' sense of smell. Our results suggest that age and smoking status affect olfaction and that cumin and sesame should be replaced by more familiar odorants when testing olfactory perception in patients. In all, this modified test for olfaction is more culturally appropriate for testing geographical region patients, in addition to being a cost-effective modality by which to evaluate smell clinically.

\section{Acknowlegdement}

We are grateful to Dicle University DUBAP for their sponsorship about English editing of this manuscript.

\section{Authorship contribution}

AG: Study design, data collection, analyses and writing

MA: Study design, data collection and writing

FEÖ: Study design and writing

BY: Data collection, analyses

EŞ: Data collection, analyses

SB: Analyses and writing

IT: Analyses and writing

\section{Conflicts of Interest}

The authors declared that there were no financial closure and conflict interests.

\section{References}

1. Hadley K, Orlandi RR, Fong KJ. Basic anatomy and physiologyof olfaction and taste. Otolaryngol Clin North Am. 2004; 37: 11151126

2. Doty $R L$, Shaman $P$, Kimmelman $C P$, Dann MS. University of Pennsylvania Smel Identification Test: a rapid quantitative olfactory function test for the clinic. Laryngoscope. 1984; 94: 176-178.

3. Fokkens WJ, Lund VJ, Mullol J, et al. European position paper on rhinosinusitis and nasal polyps 2012. Rhinol Suppl. 2012 (23): 93.

4. Mackay-Sim A, Grant L, Owen C, et al. Australian norms for a quantitative olfactory function test. J. Clin. Neurosci. 2004; 11: 874-879.

5. Cain WS, Gent JF, Goodspeed RB, Leonard G. Evaluation of olfactory dysfunction in the Connecticut Chemosensory Clinical Research Center. Laryngoscope. 1988; 98: 83-88.

6. Katotomichelakis M., Balatsouras D. Tripsianis G. et al. Normative values of olfactory function testing using the 'sniffin'sticks'. Laryngoscope. 2007: 117: 114-120.

7. Konstantinidis I, Printza A, Genetzaki S, et al. Cultural adaptation of an olfactory identification test: the Greek version of Sniffin' Sticks. Rhinology. 2008; 46: 292-296.

8. Yuan $\mathrm{BC}$, Lee $\mathrm{PL}$, Lee $\mathrm{YL}$, et al. Investigation of the Sniffin' Sticks olfactory test in Taiwan and comparison with different continents. J Chin Med Assoc. 2010; 73: 483-486.

9. Shu CH, Yuan BC. Assessment of odor identification function in Asia using a modified "Sniffin' Stick" odor identification test. Eur Arch Otorhinolaryngol. 2008; 265: 787-790.

10. Eibenstein $A$, Fioretti $A B$, Lena $C$ et al. Olfactory screening test: experience in 102 Italian subjects. Acta Otorhinolaryngol Ital.
$2005 \cdot 25: 18-22$

11. Boesveldt S, Verbaan D, Knol DL, et al. Odour identification and discrimination in Dutch adults over 45 years. Rhinology. 2008; 46, 131-136

12. Silveira-Moriyama $L$, Sirisena D, Gamage $P$ et al. Adapting the Sniffin'Sticks to diagnose Parkinson's disease in Sri Lanka. Mov Disord. 2009; 24: 1229-1233.

13. Silveira-Moriyama L, Carvalho MJ Katzenschlager $R$, et al. The use of smel identification tests in the diagnosis of Parkinson's disease in Brazil. Mov Disord. 2008; 23: 2328-2334

14. Chan A,Tam J, Murphy C, Chiu H, Lam L. Utilty of olfactory identification test for diagnosing Chinese patients with Alzheimer's disease. J Clin Exp Neuropsychol. 2002; 24: 251-259.

15. Hummel T, Sekinger B, Wolf SR, Pauli E, Kobal G. 'Sniffin' sticks': olfactory performance assessed by the combined testing of odor identification, odor discrimination and olfactory threshold. Chem Senses 1997 22: 39-52.

16. Doty RL, Shaman P, Applebaum SL, Giberson R. Siksorski L, Rosenberg L. Smell identification ability: changes with age. Science. 1984; 226: 1441-1443.

17. Hummel T, Kobal G, Gudziol H, Mackay-Sim A. Normative data for the "Sniffin' Sticks" including tests of odor identification, odor discrimination, and olfactory thresholds: an upgrade based on a group of more than 3,000 subjects. Eur Arch Otorhinolaryngol. 2007; 264: 237-243

18. Orhan KS, Karabulut B, Keleş N, Değer K Evaluation of Factors Concerning the Olfaction Using the Sniffin' Sticks Test. Otolaryngol Head Neck Surg. 2012; 146: 240246.

19. Katotomichelakis M, Balatsouras D, Tripsianis
$G$, et al. The effect of smoking on the olfactory function. Rhinology. 2007; 45: 273-280.

20. Ahlstrom R, Berglund B, Berglund U, Engen $T$, Lindvall T. A comparison of odor perception in smokers, nonsmokers, and passive smokers. Am J Otolaryngol. 1987; 8: 1-6.

21. Moncrieff RW. Smoking: its effect on the sense of smell. Am Perfumer. 1957; 60: 40-43.

22. Berglund B, Nordin S. Detectability and perceived intensity for formaldehyde in smokers and non-smokers. Chem senses 1992; 17: 291-306.

23. Woodworth RS, Schlosberg H. Experimenta Psychology. New York: Henry Holt\& Co, 1960: 317

24. Eibenstein A, Fioretti AB, Lena C, Rosati N Amabile G, Fusetti M. Modern psychophysical tests to assess olfactory function. Neurol Sci. 2005; 26: 147-155.

25. Neumann C, Tsioulos, K, Merkonidis C, Salam M, Clark A, Philpott C. Validation study of the "Sniffin' Sticks" olfactory test in a British population: a preliminary communication. Clin Otolaryngol. 2012; 37: 23-27.

Assist. Prof. Aylin Gul

Department of Otorhinolaryngology

Dicle University

School of Medicine

Diyarbakir 21280

Turkey

Tel: +90 $4122488001-4345$

Fax: +90 4122488523

E-mail: draylingul@gmail.com 